sungen als Stütze für den gegenwärtig vermuteten Elektronenwert angesehen werden. Um jedoch die Bedeutung derartiger Experimente für den Nachweis der Universalität eines Begriffes wie den des Elektrons richtig einzuschätzen, wird man nicht aus den Augen verlieren dürfen, daß bei gewissen radioaktiven Erscheinungen, wie eben beispielsweise bei der $\alpha$-Strahlung, menschliche Experimentierkunst - wenigstens bis heute - in gewissem Sinne zu Ende ist; selbst durch Aufbietung der größten uns derzeit zur Verfügung stehenden Energien, durch Anwendung höchster und tiefster Temperaturen, gewaltiger Drucke sind wir bekanntlich auBerstande, den Ablauf radioaktiver Reaktionen zu beschleunigen oder zu verzögern. Von diesem Gesichtspunkte aus scheint es nun allerdings weniger merkwürdig, wenn mit der beharrlichen RegelmäBigkeit, mit welcher radioaktive Erscheinungen unbeirrt durch äuBere Umstände ihren Ablauf nehmen, eine RegelmäBigkeit wie die der $\alpha$-Strahlung verbunden ist. Ebenso wie beispielsweise ein in der Naturseltenheit der Dobschaver Eishöhle eingeschlossener Beobachter aus der von ihm gefundenen Konstanz der Temperatur seiner Umgebung während des ganzen Jahres nicht auf eine solche auf dêr ganzen Erde sehlieBen durfte, scheint es in dieser Belenchtung vielleicht gewagt, aus einer so ausnahmsweisen und speziellen Erscheinung wie der des radioaktiven Zerfalles ein Fundament zu einer Weltanschauung von der Universalität der heutigen Form der Elektronentheorie baven zu wollen.

8 22. Die Frage nach der Existenz von Elektronen. - Die übrige grobe Zahl der verschiedensten Experimentalergebnisse konnte zwar unter Zugrundelegung eines Elementarquantums der mehrfach erwähnten Größe rechnerisch gedeutet werden, doch stehen in den einzelnen Fällen ad hoc konstruierte Hypothesen, ungelöste Schwierigkeiten, zu wenig direlkte oder genaue Beobachtungen einer Auffassung als selbständiges Argument für die Elektronenvorstellung noch weitaus mehr im Wege.

Nun scheint es ja sehr gut denkbar und vielleicht sogar wahrscheinlich, daß eine große Anzahl von Berechnungen und Experimenten, die den Sachverhalt nicht so klar überblicken lassen wie die Ehrenhaftschen, das Licht der wissenschaftlichen Welt deshalb nicht erblickten, weil sie eben im Widerspruche mit der herrschenden. Theorie gewesen wären.

$\mathrm{Ob}$ nun tatsächlich unter gewissen, spezjellen Umständen, ob nux unter diesen Umständen immer die gleichen Elektrizitätsladungen auftreten, ob Experimente und Rechnungen bereits durchgeführt, aber noch nicht veröffentlicht wurden, die sich nicht im Sinne der Elektronentheorie deuten lassen, die Lösung dieser Fragen muß der nahen Zukunft überlassen werden.

Damit eröffnet sich dem Experimentator ein reiches Arbeitsfeld mit einer Fülle von Aufgaben, denn er wird bestrebt sein, einerseits neve, mög- lichst direkte Methoden zur quantitativen Verfolgung von Erscheinungen zu finden, an welchen ein Effekt der elektrischen Elementarladung sich zeigen müßte, andrerseits die bereits bekannten Methoden auf eine Genauigkeit zu steigern, die der Tragweite daraus gezogener Schlüsse ebenbürtig ist. Falls diese Beobachtungen eine genügend stützende Grundlage für die Annahme eines elektrischen Elementarquantums bieten werden, wird es Sache der Theorie sein, das Zustandekommen in plausible Bilder zu bauen. Als bereits feststehende Tatsache muB jedoch aufgenommen werden, dab die einfachen und ungezwungenen Experimente Ehrenhafts regellos alle denkbaren Ladungen bis zum zweitausendsten Teile des bis nun unteilbar vermeinten universellen elektrischen Elementarquantes beobachten lassen, so daß von einem unveränderlich großen Elektron in der gegenwärtig verrnuteten Ladung von etwa $4,7 \cdot 10^{-10}$ e. st. E nicht die Rede sein kann.

\section{(Schlus folgt.)}

\section{Mitteilungen aus verschiedenen Gebieten der Biologie.}

Vererbungsstudien an Mäusen. (L. Plate, Arch. $\dot{f}$. Entwicklungsmechanik 44, 1918, S. $291-336$, mit einer Tafel und 5 Textabbildungen.)

Die Abhandlung beschäftigt sich mit zwei Problemen, mit der Vererbung des Zobelanflugs bei Hausmäusen als eines Beispiels einer progressiven Mutation und mit der Vererbung der WeiB-Scheckung.

1. Von den orangefarbigen Mäusen (yellows) kommt eine Varietät vór mit dunklem Rückenanflug, welcher zobelfarbig (sable) genannt wird. Dieser dunkle Anflug tritt in sehr verschiedener Stärke auf und zeigt sich bei den jungen Tieren am intensivsten, um im Laufe des Lebens mehr oder weniger abzublassen, so daB die Zobelmaus in vielen Ful. len schlieblich rein orangefarbig wird. Man unter scheidet diese Abstufungen als schwarze, mittlere und gelbe Zobelmäuse. Bei den schwarzen (szbl) sind die Haare der Zobelregion in ganzer Länge schwarz. Bei den beiden anderen Sorten haben diese Haare eine gelbe Fndbinde, und zwar ist bei den mittieren (mitzbl) die Haarbasis dunkel, bei den gelben (gezb1) hell. Eine scharfe Grenze läbt sich zwischen diesen 3 Sorten nicht ziehen, da sie durch Mauser allmählich ineinandex übergehen. Das Tempo der Aufhelling variiert individuell außerordentlich, so daB die nächst bellere Stufe in einem Zeitraum erreicht wird, welcher zwischen 1 Monat und 2 Jahren schwankt. Die Aufhellung beginnt fast immer zuerst am hinteren Körperende und sehreitet nach vorn vor. Bei 5 Individuen wurde eine nachträgliche Verdunkelung beobachtet, indem das schon aufgehellte oder bei der Geburt helle Tier allmählich wieder dunkler wurde; bei einem Exemplar hellte sich später diase zweite Verdunkelung wieder auf. Die Rückbildung des Pigments geht besonders rasch vor sich, wenn die Tiere die Faktoren $\mathrm{Ee}^{\mathfrak{t}}$ ) enthalten, und zuweilen zeigen sofche Mäuse schon

1) E bedingt schwarze Augen und dunkle Haariar. bung, e rote Augen und hellere Färbung. Vgl. L. Plate Vererbungslehre, Leipzig, W. Engelmann 1913 , S. $129 \mathrm{ff}$. 
bei der Geburt keinen Anflug and sind voll orangerarbigen nicht $z u$ unterscheiden. Es beruht dies auf dem pigmenthemmenden Einfluß von e, welcher beson ders deutlich darin zum Ausdruck kommt, dab die rotiugrigen ee-Mäuse nie einen Zobelanflag besitzen. Eine Ausnahme hiervon ist mir nur zheimal begegnet. Lbensowenig wie orangefarbige Mäuse ( $Y$. . .) aus nieht-orangefarbigen (yy ...) lervorgehen können, ebensowenig können Zobelmäuse $\left(\mathrm{Y}^{\prime} \mathrm{Y} . ..\right)$ von NichtZobelmäusen geworfen werden. Eine junge Maus kann aber $Y^{\prime}$ enthalten und iuBerlich doch keinen Zobelanflug besitzen, entweder weil derselbe schon in selir jugendlichem Alter wieder sich rückgebildet hat, oder weil er infolge von ee uberhaupt nicht zur Latfaltung kam. Die Zobelmäuse sind als eine Varietät der orangefarbigen anzusehen, indem $Y$ eich mutativ in $\mathrm{T}^{\prime}$ verwandelt. Dies geht daraus hervor, daß die Zobelmuluse ebenso wie die orangefarbigen immer heterozygot sind und im Laufe ihres Lebens durch Aufhellung zu orangefarbigen werden. Wahhend $Y$ die Pigment. bildung durch B (Falktor für schwarzes Pigment) unterdrükt, vermag $Y$ ' dies nicht zu tun. Daher der dunkle Rückenanflug. $\mathrm{Y}^{\prime}$ ist also als ein geschwächtes $\mathrm{Y}$ anzusehen. Hieraus folgt auch, daß alle Zobelmäuse $B$ enthalten. Die schwarzen Zobelmäuse sind $Y^{\prime} y B B E E$ bzw. Ee, die mittleren und gelben $Y^{\prime} y B b E E$ bzw. Ee. Da $Y^{\prime}$ in dem Grade señer Schwächung sehr variert, sehen zuweilen die $Y^{\prime} y \mathrm{Bb}$ als junge Tiere wie szbl aus. Da nur die Heterozygoten lebensfahig gind, kommt kein Tier von der Zusammensetzung $\mathrm{YY}$ ' vor. Man kann also auch nicht behaupten, da $\mathrm{B}$ uber $\mathrm{Y}^{\prime}$ dominiert, oder vmgekehrt $Y^{\prime}$ iber $Y$. Die zahlreichen Kreuzungen stimmen mit diesen Auffassungen überein, da die beobachteten und erwarteten Sorten harmonieren. Man kann also auch nicht $\mathrm{Y}^{\prime}$ dadurch erzielen, das man echte orangefarbige Mäuse dureh mehrere Generationen hindurch mit schwarzen Mäusen kreuzt. Obwohl Y' dem $Y$ sehr nahe steht, ist es doch als eine echte Erbeinheit anzusehen, welche frellich, wie alles Organische, in ihrer Wirkung und daher auch wohl in ihrer chemisch-physikalisehen Konstitution variiert. Ts wurde nichts beobachtet, was für "Gameten-Unreinheit" oder dafür spricht, dah ein Faktor durch Zusammentreifen mit einem andern dauernd verundert werden kann. Die Umwandling von $Y$ in $Y^{\prime}$ ist als Beispiel einer progressiven Mutation anzusehen.

2. Die WeiB-Scheekung der Mänse int ein rezessiven Merkmal gegentiber der Einfarbigkeit, welche in der Regel vollkommen dominant ist. In einzelnen Fillen sind die Feterozygoten schon äußerlich kenntlich (Zeatypus). Die Weis-Scheckung hat mit Albinismus nichts zu tun und kann daher nicht, oder nur in ungenaver Ausdrucksweise als partieller Albinismus bezeichnet werden. Die grobe Variabilitat der WeilScheckung laBt sich exklären, wenn man auber dem allelomorphen Paar $\mathrm{S}=$ Einfarbigkeit, $\mathrm{s}=$ Scheckung, eine Anzahl yon gleichsinnigen Erbfaktoren annimmt, deren Fehlen die Variabilität erzeugt. Fehlt nur einer, so entsteht der erste Seheckungsgrad, fehlen 2 der zweite usw. Die Beobachtungen lassen auf mindestens 7 oder 8 solcher Modifikatoren sehlieben, wabrscheinlich aber ist ibre Zahl noch größer. Auf dem Boden dieser Theorie der Entstehung der Schecking dureh polymere Faktoren wird es verständlich, dab gescheckte Tiere einfarbige Junge werfen können, daf die $F_{ \pm}$zuweilen iiber den Scheckungsgrad der Eltern transgredieren, das an jeder Stufe eine Selektion nach zunehmender oder abnehmender Pigmentierung (Guénot, Castle) ausgefuht werden kann. 1. a. Alle beobachteten Thtsachen werden auf diese Weise ohue Annahme einer rnkon. stanz der Erbfaktorea oder einer Gameten-Tnreinheit auf streng mendelistischer Basis verständlich. Der niedere Grad der Scheckung dominiert nicht uber den höheren, sonderu die verschiedenen Grade siud Glieder ciner polygenetischen Reihe. Durhams und Hagedooms Angabe, dar es eine dominarite Scheckung gibt, ist nock zweifelhaft. Autoreferat.

Oberflächenspannungsdifferenzen als eine Ursache der Zellteilung. (Josef Spek, Arohiv für Entwicklungsmechanit d. Org. Bd. 44, Heft 1.1918, S. 5-113.)

Schon im Jahre 1876 sprach $O$. Butschli die Vermutung ans, daß Oberflächenspannungsdifferenzen am Zelleib die Ursache der Zellteilung seien. Der Beweis hierfür wurde nun dadurch ermöglicht, daß Oberflächenspannungsänderungen an toten Flüssigkeitstropfen wie an lebenden Zellen stets ron ganz gesetzmäßigen Erscheinungen begleitet sind. Die Untersuchungen nurden dadurch auf besonders sichere Grundlage gestellt, dab es gelang, eine regelmäbige Durchschnürung von Flüssigkeitstropfen durch Oberflachenspannungsdifferenzen anch künstlich am physikalischen Modell auszuführen. Ein viskoser Öltropfen flacht sich am Aquator $a b$, kerbt sich ein und schnüt sieh schließlich. ganz durch, wenn seine Aquatorzone eine Region höherër Oberilabchenspannung wird, wähend die Pole Stellen verminderter Oberflächenspannung sind. Lokale Verminderungen der Oberflächenspannung können am öltropfen durch Verseifung seiner Oberflache herbeigefüht werden. - Die Durchschnürung des Tropfens ist nun stets begleitet von typischen Strömungserschejnungen. Stets bewegt sich ein ,oberflachlicher $Z_{\mathrm{i}} \mathrm{u}$ strom" zu der Zone erhöhter Oberflïchenspannung und biegt im Xquator in das Innere der Zelle ein. Gegen die Pole, die Stellen der Spannungsverminderung, geht ein, axialer Zustrom"s, der an der Oberfläche angelangt, in eínen ,oberflächlichen Ausbreitungsstrom" ïbergeht. Dieser typische Strömungsverlauf labt sich in allen Einzelheiten auch bei der gleichmäBigen Durchschnürung der Eizellen kleiner Nematoden erkennen. Bei einseitiger Einschnürung des Zelleibes erfährt er eine charakteristisehe Abünderung, die aueh den theoretischen Erwartungen entspricht, wenn die Einkerbungsstelle eine absolat erhohte Oberflachenspanning besitzt. - Lebhaftere Strömungen während der Zellteilung lassen sich nur an günstigen Objekten beobachten, sehr häufig kommen hingegen ganz typische Verlagerungen des Zellinhaltes (Dotterkörner, Pigment!) beí Zellteílungen vor, die zich auf Plasmaströmungen zurücktuiliren lassen, die wieder ganz den theoretischen Vorstellungen entsprechen. - In besonders eindeutiger Weise ergibt sich die Abschuürung der Richtungskörper als eine Wirkung von Oberilachenspannungsdifferenzen. Die Richtungskörper entstehen, indem sich eine kleine, eng und scharf begrenzte Zone verminderter Oberflächenspannung an der Eizelle vorwölbt und sich nacher an ihrer Basislinie, einer zone erhohter Oberfluchenspannung, abschnürt. - Eine Rehe von Erscheinungen, mit deren genauerem Studium dex Verfusser gegenwartig beschaftigt ist, spricht dafür daß sich die Zellkolloide whbrend der Zellteilung in einem wasserrejeheren, verflussigteren Zustand befinden. Da das wohl auch für die Kolloide etwaiger Zellmembranen gilt, ist jedenfalls auch für Zellen, die nicht wie die Eizellen .,nackt". sind, die Möglichkeit der Entstehung von Oberilhehenspannungsdifferenzen bei der Zellteilung gegeben.

Autoreferat: 
Differenzen im Quellungsaustand der Plasmakolloide als eine Ursache der Gastrulainvagination, sowie der Einstülpungen and Faltungen von Zellplatten überhaupt. (Josef Spek, Kolloidchemische Beihefte Bd. IX, Heft 10-12, S. 259-400.) Die Einstuilpung einer Gastrula kommt nach $O$. Bütschli dadurch zustande, daB die eine (später konvex werdende) Fläche der Entodermzone sich stärker ausdehnt als die äuBere. Fs ist nun sehr wahrscheinlich, das diese stäkere Aubdelnung ter einen Fläche der sich einkrümmenden Zellplatte der Gastrula und ebenso auch bel den andern in den ersten Stadien der Ontogenie der Tiev'e eine so grobe Rolle spielenden Einstülpungs und Faltungsprozessen in vielen Fullen dureh reine Wasseraufnahme zustandekommt. Dieser Überlegung entsprechend wurden die Grundlage zu neuen Studien über diesen Gegen. stand Versuche mit Modellen, an denen die Einstülpungen usw:"ausschließlich durch einseitjg stärkere Wasseraufnalıme herbeigefübrt wurden. Stellt man sich z. B. - als Modell einer Blastula - aus Gelatineund Agargallerte eine Hohlkugel her, an der die eine Hälite (,Entodermbälfte") aus einer stärker quellbaren inneren und einer sehwächer quellbaren äuBeren Lamelle aufgebaut ist, und setzt sie ins Wasser, so stülpt sich in 1-2 Tagen das "Entoderm" durch stärkere Aufquellung seiner Blastoodelfäche selbstätig nach innen ein. In ahnlicher Weise läbt sich die Entstehung von Ausstilpungen an Hohlzylindern (z. B. Leberausstülpung) oder yon Längsfalten (Medullarrinne, Chordafalte usw.!) im Modell vorfuhren. - Daß nun bei den genannten Prozessen auch in den Organismen Quellungsenscheinungen eine Rolle spielen, wird schon dadurch wahrscheinlich gemacht, dabs die betreffenden Organanlagen sich sehr häufig durch einer besonderen Reichtum an gut quellbaren Substanzen auszeichnen. Fernerhin spricht dann aber noh ganz besonders der Umstand dafür, daB sich die Finstulpungsprozesse in weitem Maßé durch Stoffe beeinflussen; $d$. $b$. hemmen oder gar umkebren lassen, die einen mäehtigen EinfluB auf die Quellung von Kolloiden ausuben. Es sind dies besonders Salze und Säuren. In Verwertung einer ganzen Reihe von zum Teil selbst ermittelten Resultaten der Kolloidchemie der Quellung wird gezeigt, daß sich alle die intereseanten Fälle, in denen Curt Herbst durch bestimmte Salzwirkungen (Zusatz von Lithiumsaizen, Kaliumrhodamid oder Natriumbutyrat zum Seewasser, durch Weglassen der Sul fate oder des Magnesiums aus demselben) ror Jahren Exogastrulation der Seeigellarven (Gastrulen mit nach auben gestulptem Urdarm) erzielen konnte, diese dadurch zustande kommen dürte, daß Ionen, die die Quellung stark fördern, sich in der äuBeren Halfte der Zellen der Entodermregion anreichern, oder aber daf entquellend wirkende Tonen daratis entfernt werden. Das häufige Auftreten von starken Zellwucherungen an den soinst dureh Faltung oder Ausstitlpung entstehenden Organanlagen wind auf Grund gewisser Beobachtungstatsachen auf deren Reichtum an stark quellbaren Substanzen zurückgefährt. Autoreferat.

Delegierte der neutralen Staaten, die an der internationalen Meeresforschung teilmehmen: Schweden, Norwegen, Holland und Dänemark, haben rom $23,-25$. Mai in Kopenbagen verschiedene fischereiokonomische, biologische und hydrographische Fragen behandelt. Von den fischereiökonomischen Fragen ist besonders zu nennen die Annahme von Vorschlägen zu umiassenden Untersuchungen des Fischbestandes in der Nordsee nach dern Kriege. Man wïnsehte ansfülurliches Ma- terial hierüber, um es mit dem großen aus der Zeit vor dem Kriege gesammelten Material zu vergleichen. Das ist von großer Bedeutung für die Feststellung künftiger Schonmaßregein, besonders für Plattîische und Sehellfische. - Dr. Redeke (Holland) und Dr. Johansen (Dänemark) machten interesiante. Mitteilungen ilber den bis jetzt beobachteten Wechsel des Fischbestandes in der Nordsee während des Krieges. Selbstverstandlich konnte man solche Untersuclungen nur in kleinem Maßstabe austiubren und nur in Küstengewässern, bat also entscheidende Resultate noch nicht erreicht. Aber es ist doch von großem Interesse, besonders durch die holländischen Beobachtungen iber die Scholle festgestellt zu laben, daß schon zwei Jahre nach dem Ausbruch des Krieges eine Erhöhung der Durchschnittsgröße bemerkbar geworden ist im Vergleich mit der Durchschnittsgröbe vor dem Kriege. Auch die Anzahl der Schollen hat sich erheblich vergröbert. Das ist für Holland in den jetzigen Ernährungsverhälnissen sehr nützlich gewesen. Nach dem Kriege wird es für die internationale Meeresfonsching eine große und bedeatungsvolle Aufgabe sein, diese Fragen zu erforschen und mit den von der modernen Meeresforschung viele Jahre gepruiten Methoden zuverlässige Angaben herbeizuschafien. Professor Gran, Delegierter für Norwegen, hielt einen sehr instruktiven Vortrag iber die Plankton-Produktion des Kristianiafjords. Er wird von dem internationalen. Bureau herausgegeben werden. Dr. Hjort, ebenfalls norwegischer Delegierter, beteiligte wich an der Diskussion der meisten vorliegenden Fragen. Besonders bemerkenswert ist ein Vortrag über einige seiner Untersuchungen über den Hering in kanadischen Gewässern, die er während seines Aufenthalts in Kanada für die kanadische Regierung ausgefüht hat. Er wies anf Ahnlichkeiten von bemerkenswerter Art zwischen den Altersstufen des Herings im atlantischen Meere und in kanadischen und norwegischen Gewärsern hin. - Der Chet des Bureaus für Fischereiadministration in Scliweden, Dr. Nordquist, berichtete uber einigue Ergebnisse der nelieren Untersuchungen von Dr. Andersson über die Rassen und Wanderungen des Herings in Kattegat und Skagerak.

$\checkmark$ iel Zeit beanspruchte eine Diskussion über ein großes hydrographisches, aus dem atlantisehen. Meero gewonnenes Material, namentlich deutsehes, hollandi sches und dänisches. Dieses Material ist wärend des Krieges auf Betreiben de Präsidenten Professor Peters son bei dem internationalen Bureau bearbeitet worden. Der hollïndische Delegierte, Professor tan Everdingen, und der Direktor des meteorologischen Instituts in Kopenhagen. Kapitän Ryder, nebst Profossor Pelters son ans Schweden, Professor Knudsen und Dr. Gehrke aus Dänemark nahmen an der Diskuesion dieser Fragen teil. Der Generalsekretär, Kommandör Drechsel, legte ein Schreiben an die an der internationalen Meeresforschung teilnehmenden Regierungen vor, über die Notwendigkeit, diese Forsehungen so bald wie möglich nach dem Kriege wieder aufzunehmen, Schließlich wurde ein Vorschlag zum Budget für das nächste Finanzjahr angenommen, das mit verschiedenen anderen Vorschligen an die Delegierten derjenigen kriegtihrenden Lilnder gesandt werden wird, die an der internationalen Meeres. forschung teilnehmen.

Drechsel, Kopenhagen.

Uber den Einflus von Kontaktreizen und mechanischem Reiben auf das Wachstum und den Turgeszenzzustand von Keimstengeln. ( $P$. Stark, Ber. $d . D$. Bot. Ges. 35, 1917.) Wenn man Keimstengel der ver 
sehiedensten Pflanzenarten mit einem Korkstäbchen wiederholt reibt, dann kann man folgende Erscheinung beobachten: Die Spitze beginnt sich allmählich nach der geriebenen Seite überzuneigen, die Krümmung chreitet mehr und mehr nach basaleren Regionen fort, und schließlich sinkt das Pflämzchen vollständig erschlafft zur Erde nieder. In diesem Zustande kana es längere Zeit verharren. Dann aber erfolgt wieder ein Aufrichten, und alle Phasen werden in umgekehrter Richtung durchlaufen, bis der Keimling wieder voll ständig straff und aufrecht dasteht. Kausal ist das Umsinken dadurch bedingt, daB durch das Reiben die Transpiration ganz erheblich erhoht wird und infolgedessen ein so starker Wasserverlust eintritt, das die Gewebespannung nicht mehr ausreicht, das Pflänzchen aufrecht zu erhalten. Das läßt sich durch Wägung einwandfrei feststellen. Es ergibt sich dabei eine Transpirationssteigerung um $100 \%$. Aber anch auf anderem Wege läßt sich die verstärkte Wasserabgabe ermitteln. Man braucht nur den Stengel mit einem Streifchen getrockneten Kobaltpapiers zu umwickeln. Kobaltchlorür hat nämlich in trockenem Zustande eine intensiv blaue Farbe, rötet sich aber sofort bei der geringsten Wasseraufnahme. Es zeigt sich nun, dafs der Papierstreifen nach küzester Frist an der Stelle, die gerieben wurde, einen roten Strich aufweist. Diese Erscheinung liefert uns eine Erklärung für die Ursaehen der Transpirationszunalume. Die Keimstengel der-Versuchspflanzen sind, um eine schädigende Wasserabgabe $z u$ verhindern, mit einer Wachsschicht tiberzogen, und dieser Schutzpanzer wird durch das Reiben partiell abgestreift. Die Pflanze besitzt aber das Vermögen, den Schaden wieder auszubessern, und darauf beruht $e s, d a B$ die Keimlinge am folgenden Tage wieder aufrecht dastehèn. Interessant ist nun das Verhalten you Keimlingen, die man sofort nach dem Reiben in den dampigesättigten Raum stellt. Da in diesem Fall die Transpiration fast vollständig sistiert wird, so tritt keine Reaktion ein. Dagegen kann das Umsinken sofort erzielt werden, wenn man die Objekte in trockeno Atmosphäre verbringt. Bemerkenswert ist nun, daß ein solches verspätetes Umsinken auch dann erfolgt, wenn sich die geriebenen Keimlinge 3-4 Tage im Dampiraum befunden hatten. In dieser Zeit haben Pflanzen, die in trockener Luft stehen, ihre Wachsschicht längst regeneriert. Daraus folgt, daß die Neubildung der Sehutaschicht blok dann stattfindet, wenn sie biologisch notwendig ist, nicht aber, wenn die Dampfsättigung ein Welken ausschliebt. Dies läBt sich auch aus folgender Tatsache ersehen: LäBt man Keimlinge vollständig im dampfgesätigten Raum aufwachsen und bringt sie dann plotzlich in eine trockene Atmosphäre, dann beginnen sie vielfach schlafí nieder. zusinken, ohne dals sie gerieben wurden. Hier ist eben unter den veränderten Bedingungen die Wachsbildung primär unterblieben. Sie tritt erst nachträglich bei längerem Aufenthalt im trockenen Ram ein, wobei aich dann die Keimlinge wieder anfrichten. Wir haben es hier mit zweckmäßigen Reaktionen zu tun, die sicher in der freien Natur eine große Rolle spielen. Es ist ja eine bekannte Tatsache, daß bei vielen Pflanzen die Wachsbildung mit den Standorteverhältnissen im Zusammenhang steht und. je nach der Vegetationsperiode geändert werden kann.

P. St.

Zur Kenntnis der phototaktischen Richtungsbewegungen (J. Buder, Jahrb. f. wiss. Bot. 58, 1917). Die phototaktischen Reaktionen der Mikroorganimen sind von jeher ein beliebtes Studienobjekt für Zoologen und Botaniker gewesen. Dite Arbeit von Buder liefert nun neben einer kritisehen Zusammenstellung der Literatur auch eine Reihe sehx bemerkenswerter neuer Beobachtungen, die an Chlamydomonaden, Flagellatea und anderen, an der Grenze von Tier und Pflanzenretch stehenden Organismen gesammelt wurden. Besonderes Interesse verdienen die Versuche, bei denen zwei yeyschieden gerichtete Strahlenbündel gleichzeitig auf die Versuchsobjekte einwirkten. Wahrend sich die Organiemen bei einfacher einseitiger Beleuchtung der Lichtquelle direkt nähern oder-falls negative Phototaxis vorliegtsich von ihr entfernen, halten sle jetzt, voransgesetzt, da $B$ die beiden Lichtquellen gleich intensiv sind, die Winkel. halbierende ein. Die Bahn fallt also nicht mehr mit der Strahlenrichtung zusammen." Sind die beiden lichtquellen aber verschieden stark, dann erfolgt eine Ablenkung nach der stärkeren von den beiden, und die Richtung wird durch das Parallelogramm der Kräfte bestimmt. Der Organismus bewegt sich also so vorwärts, daß beiden Flanken dieselbe Lichtmenge pro Zeiteinheit zufliebt. Dieses "Resultantengesetz" gilt allem Anschein nach auch bei der grleichzeitigen Anwendung von drei Strahlenquellen, und sein Geltungtsbereich enstreckt sich auch auf die phototropischen Reaktionen der höhren Pllanzen. Da diese festgewachsen sind, so beantworten sie den Reiz nicht mit einer Ortsveränderung, sondern sie krimmen sich nur in die durch das Resultantengesetz gegebene Richtung ein. Eingehende Daten existieren hier allerdings noch nicht, doch gibt es schon deutliche Hinweise "darauf, daß auch hier - und ebenso beim Zusammenwirken von Schworkratt und Zentrifugalkraft - dieselben GesetzmüBigkeiten herrschen. $P$. St.

Zur Verwertung der Pilze (Raebiger: Zeitsehr. $t$. Fleisch- und Milchhygiene 1\%, 1917). Wie bekannt ist, machen die Giftpilze nur einen sehr geringen Teil unserer Pilabestände aus. Das Kaiserliche Gesundheitsamt macht in einem Merkblatt blos 11 Arten nambaft. Aber axch von diesen kann eigentlich blok der. Knollenblatterschwamm (Amanita phalloides und A. mappa) als absolut gefährlich bezeichnet werden. Alle iibrigen Pilze werden da und dort mit gewissen Vorsichtsmakregeln als Speisepilze verwertet. So verlieren der Pantherpilz (A. pantherina), der Perlschwamm (A. rubescens), ja sogar der Fliegenpilz (A. muscaria) nach Abziehen der Haut ihre Giftigkeit. Der Speiteufel (Russula emetica), der Satanspilz (Boletus satanas) und die Giftmorchel (Gyromitra essulenta) werden durch das Abkochen genieBbar. Der Kartoffelbovist (Scleroderma vulgare) wirkt nur in älteren Exemplaren und in grober Menge genossen sehadlich. Der falsche Pfifferling endlich (Cantharellus aurantiacus) und der Sehwefelkopf (Hypholoma fasciculare) gelangten blos durch ihren bitteren Geschmack in den Ruf der Giftigkeit. Wenn diese Formen aich auch gröbtenteils fir die menschliche Nahrung nicht emptehlen, so stellen sie doch ein recht nütaliches Virhfutter dar, das besonders in Vermisehung mit auderen Arten keinerlei Gefährdung bietet. Tatsäehlich werden diese Arten vielfach spontan von Wild- and Weidetieren aufgesucht, und sie empfehlen sich - besonders unter den gegenwärtigen Verbältnissen - geradezu als Kraftfutter, weil die Trockensubstanz neben Kohlehydraten mehr Eiweib enthält als selbst Fleisch. Nach einer Berechnung wîrden sich bei viermaliger Frnte im Jahr auf deutschem Boden 2000 Millionen Kilograman Pilzsubstanz gewinnen lassen. Das entspricht einem EiweiBgehalt von ca. 50 Millionen Kilogramm. P. St. 